\title{
Crystalline stromal dystrophy: histochemistry and ultrastructure of the cornea
}

\author{
R. O. WELLER AND F. C. RODGER \\ From the Department of Pathology (Neuropathology), Southampton University, and the \\ Department of Ophthalmology, Princess Margaret Hospital, Swindon
}

SUMMARY A report of a woman in her fifties with Schnyder's crystalline stromal dystrophy is presented. There is no personal or family history of hypercholesterolaemia. A full thickness corneal disc was removed before grafting and examined by polarised light, histochemistry, and electron microscopy. Specific lipid histochemical methods showed deposits of cholesterol and cholesterol ester in the superficial stroma and in Bowman's membrane, but no triglyceride or free fatty acids were detected. Electron microscopy showed disruption of the superficial corneal stroma and Bowman's zone by cholesterol crystals and the presence of extracellular lamellated lipid droplets with a 4-4.5 $\mathrm{nm}$ periodicity. The epithelium, endothelium, and Descemet's membrane were virtually unaffected. The role of corneal grafting in crystalline stromal dystrophy is evaluated and the possible mechanisms of lipid accumulation in this disease are discussed.

Crystalline corneal dystrophy was first described by van Went and Wibaut in 1924 and by Schnyder in 1927 (Delleman and Winkelman, 1968). Many cases are familial with a dominant inheritance (Delleman and Winkelman, 1968), but there are accounts of isolated cases where a family history is not established-for example, patients reported by Bonnet et al. (1934) and Gillespie and Covelli (1963). The main features of the dystrophy (Delleman and Winkelman, 1968) consist of bilateral subepithelial corneal opacification situated immediately posterior to Bowman's membrane and central in position; numerous crystals are also seen in the affected central zone of the cornea, but there is no inflammation or irritation. A white girdle is often seen at the limbus, and some cases are associated with familial hyperlipoproteinaemia (Bron et al., 1972). The condition is frequently present from early youth and rarely requires corneal grafting.

The present paper concerns a patient with crystalline stromal dystrophy without a firm family history. A full-thickness corneal graft was performed, which has allowed a more complete ultrastructural and histochemical examination of the cornea in this disease than has previously been published (Garner

Correspondence to Prof R. O. Weller, Department of Pathology (Neuropathology), Level E, South Laboratory and Pathology Block, Southampton General Hospital, Southampton SO9 4XY. and Tripathi, 1972; Babel et al., 1973; Ghosh and McCulloch, 1977).

\section{Case history}

The patient is an unmarried woman now in her fifties who presented 10 years ago with progressive clouding of the central portion of both corneae (Fig. 1). Stromal clouding was also observed outside the central area of the cornea, but this is not well shown in Fig. 1. A marked arcus lipidalis was seen in both eyes. Apart from hirsuties no other clinical abnormality was detected. All living members of the patient's family were contacted, and none has diabetes mellitus or any corneal disorder.

\section{CLINICAL INVESTIGATIONS}

The patient's fasting blood sugar levels over the 10-year period were never above the normal range. Similarly, her fasting blood lipid levels were below $6.5 \mathrm{mmol} / 1$ on all occasions except one. In this instance the level was $7 \cdot 2 \mathrm{mmol} / \mathrm{l}$, but there is some doubt about whether it was a fasting level.

Fasting blood lipid levels were obtained from all living members of the patient's family (Table 1). All the sera were clear, but 1 fasting serum cholesterol level was somewhat raised; the triglyceride level in this case was normal. Two relatives had raised fasting triglyceride levels. The patient's 

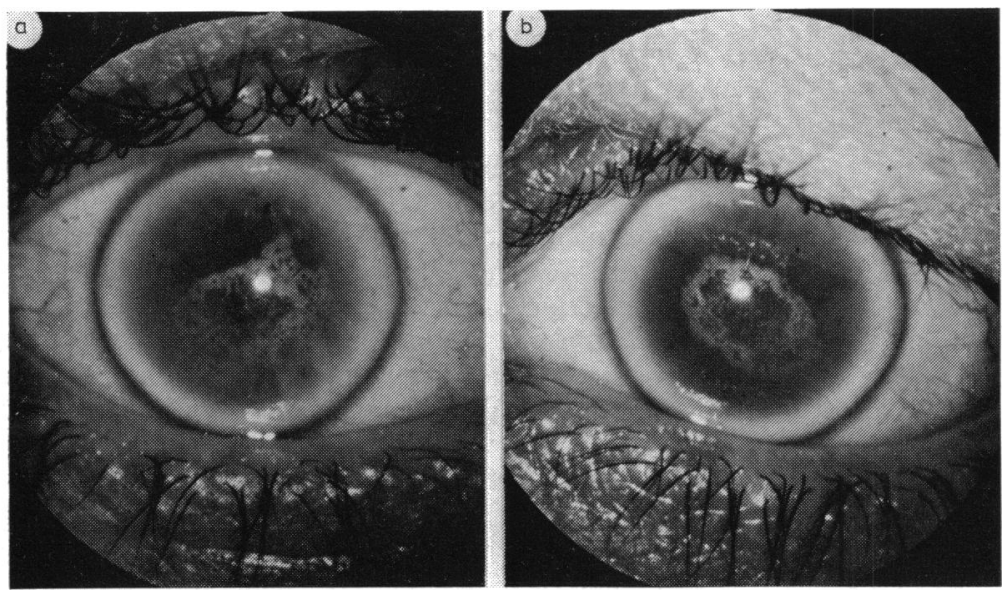

Table 1 Fasting blood lipid levels of extant close relatives of the patient

\begin{tabular}{lll}
\hline & $\begin{array}{l}\text { Fasting serum } \\
\text { cholesterol in } \\
\text { mmol/l } \\
(\text { normal 4.0-8.2) }\end{array}$ & $\begin{array}{l}\text { Fasting serum } \\
\text { triglycerides in } \\
\text { mmol/l } \\
(\text { normal } 0 \cdot 79-1 \cdot 69)\end{array}$ \\
\hline Brother & $7 \cdot 5$ & $3 \cdot 2$ \\
1st Cousin (mother's side) & $5 \cdot 7$ & 0.79 \\
1st Cousin (mother's side) & 6.0 & 1.60 \\
1st Cousin (mother's side) & $4 \cdot 2$ & 1.31 \\
1st Cousin (mother's side) & $5 \cdot 7$ & 0.79 \\
Aunt (father's side) & $7 \cdot 0$ & 1.8 \\
1st Cousin (father's side) & 8.3 & 1.4 \\
\hline
\end{tabular}

brother's level was markedly raised and her aunt's level minimally; in the aunt the cholesterol level was normal. These results show no association of the patient's crystalline stromal dystrophy with familial hyperlipoproteinaemia.

CORNEAL GRAFTING

Corneal grafting has been attempted in very few cases of crystalline stromal dystrophy, for, despite the obvious corneal lesion, reasonable vision is maintained for the greater part of the patient's life. It is difficult from the small number of grafts that have been performed, and from their brief follow-up, to assess the value of this procedure. In the present case the penetrating keratoplasty was successfully carried out in October 1977, as the patient was no longer able to continue her job. The operation was successful and uncomplicated, but 1 month after the operation there was evidence of subepithelial opacity in the cornea. Then the patient had an unfortunate fall in the street from which she sus- tained epithelial abrasion of the graft, which healed only with the aid of a soft contact lens after readmission to hospital. There appears to be some permanent stromal damage 7 months after grafting.

\section{PATHOLOGY}

The $8 \mathrm{~mm}$ diameter full thickness corneal disc removed prior to grafting was fixed in 3\% glutaraldehyde in $0 \cdot 1 \mathrm{M}$ cacodylate buffer for $4 \mathrm{hr}$. After 3 days' washing in $0.1 \mathrm{M}$ cacodylate buffer containing $7.5 \%$ sucrose at $\mathrm{pH} 7.4$ strips of cornea, including the central cloudy area, were taken for light microscopy, lipid histochemistry, and electron microscopy. Some strips were embedded in paraffin and $6 \mu \mathrm{m}$ sections cut and stained with haematoxylin and eosin, haematoxylin van Gieson, periodic acid Schiff, and diastase PAS. Tissue for electron microscopy was postfixed in Millonig's osmium tetroxide, dehydrated, stained with uranyl acetate, and embedded in Spurr resin. Sections $1 \mu \mathrm{m}$ thick were stained with $1 \%$ toluidine blue, and ultrathin sections were cut, stained with lead citrate, and examined in a Phillips 300 electron microscope.

Frozen sections of corneal strips were cut for lipid histochemistry. They were stained by the oil-red-O and perchloric acid naphthoquinone (PAN) method for cholesterol (Adams, 1961), the digitonide PAN technique (Schnabel, 1964), the lipase-calcium method for triglyceride (Adams et al., 1966), and the Holczinger copper rubeanic acid method for free fatty acids (Adams, 1965). Unstained frozen sections were also prepared for examination in polarised light.

\section{Results}

HISTOLOGY OF THE CORNEA

The corneal epithelium was intact and there was 


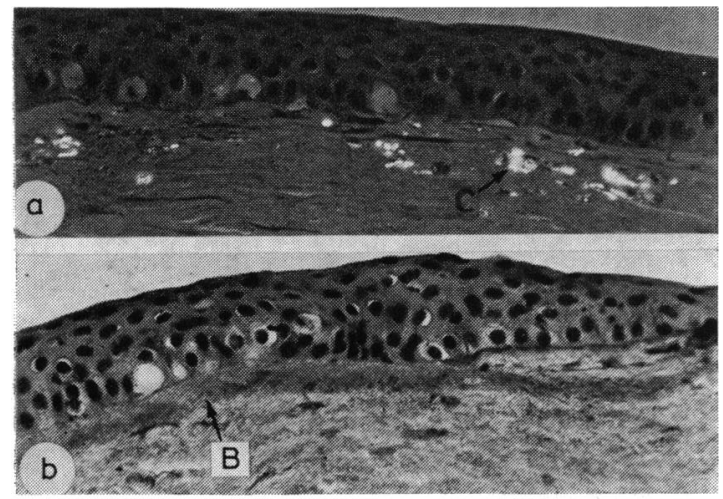

Fig. 2 Frozen section of cornea showing: (a) birefringent crystals (C) in the superficial corneal stroma. Polarised light counterstained with haematoxylin. $\times 255$. (b) High lipid content of Bowman's membrance (B) and lipid droplets in the stroma. Oil-red-O and haematoxylin. $\times 255$

no pannus. Bowman's membrane was continuous, but some disruption of the stroma was seen just deep to Bowman's membrane. No vascularisation or inflammation was observed within the corneal stroma, and neither Descemet's membrane nor the corneal endothelium showed any abnormality.

\section{POLARISED LIGHT}

Examination of frozen sections of the cornea by polarised light revealed focal clusters of needle-like crystals just deep to Bowman's membrane (Fig. 2). The crystals retained their needle-like shape at $37^{\circ} \mathrm{C}$ and $60^{\circ} \mathrm{C}$, which suggests that their melting point was above $60^{\circ} \mathrm{C}$. At $37^{\circ} \mathrm{C}$ birefringent droplets were seen in association with the crystals. These liquid crystalline droplets had a negative sign when viewed in polarised light and a first-order-red quartz plate.

\section{LIPID HISTOCHEMISTRY}

In frozen sections stained with oil-red-O Bowman's membrane was stained red or pink for much of its length, indicating a high lipid content (Fig. 2). Numerous small oil-red-O positive droplets were present throughout the stroma of the cornea but were concentrated particularly in the superficial areas of the stroma. Lipid droplets were not seen in Descemet's membrane. Sections stained by the perchloric acid naphthoquinone (PAN) and digitonide PAN methods for cholesterol and cholesterol esters showed that the lipid droplets within the stroma of the cornea were mainly cholesterol ester. No staining was seen with the lipase-calcium method for triglyceride or the copper-rubeanic acid method for free fatty acids.

\section{ELECTRON MICROSCOPY}

Epithelium. The epithelium was composed of layers of cuboidal cells with desmosomes (maculae adherentes) between adjacent cells. Occasional unattached cells were seen within the epithelium and appeared to be macrophages. The epithelial basement membrane was intact, but a few cellular processes extended into the superficial layers of Bowman's membrane.

Bowman's membrane. Bowman's membrane was recognised as a $10-12 \mu \mathrm{m}$ wide layer of collagen bundles which were smaller and less well orientated than collagen in the stroma (Fig. 3). In the present case Bowman's membrane was considerably disrupted by the presence of numerous holes separating the collagen fibres. Many of the holes were roughly oblong with rounded corners and their long axes orientated parallel to the surface of the cornea. Some of the oblong holes were $1 \mu \mathrm{m}$ or more in width. Vacuoles with circular or elliptical profiles were distributed fairly evenly throughout Bowman's membrane; many contained multilamellar bodies with a periodicity of 4-4.5 $\mathrm{nm}$.

Stroma. Aggregations of oblong-shaped spaces with rounded corners were seen in the superficial layers of the stroma. These spaces were larger than those seen in Bowman's membrane but were similar in shape. They corresponded in position to the crystalline structures seen in polarised light and were almost certainly the spaces left by the cholesterol crystals dissolved out of the tissue during preparation. Many of the spaces were long and narrow but others were short and wide (Fig. 3). Bands of collagen swept around the oblong holes, which were not bounded by membrane but only by the collagen fibrils. Multilamellar bodies were often closely associated with the geometrical holes (Fig. 4), but they were also seen throughout the stroma, always surrounded by collagen and exhibiting a $4-4.5 \mathrm{~nm}$ periodicity (Fig. 5). The oblong holes were observed only in the superficial regions of the stroma.

Endothelium and Descemet's membrane. Multilamellar bodies were seen in the stroma at the edge of Descemet's membrane but did not extend into it (Fig. 6). Descemet's membrane itself had some dark granular material deposited along its stromal aspect, but the rest of the membrane showed a diffuse grey electron density (Fig. 6). The endothelium was complete, and, although it was not optimally fixed, no structural abnormality was detected.

\section{Discussion}

The case of crystalline stromal dystrophy of the cornea presented here has no firm family history of 


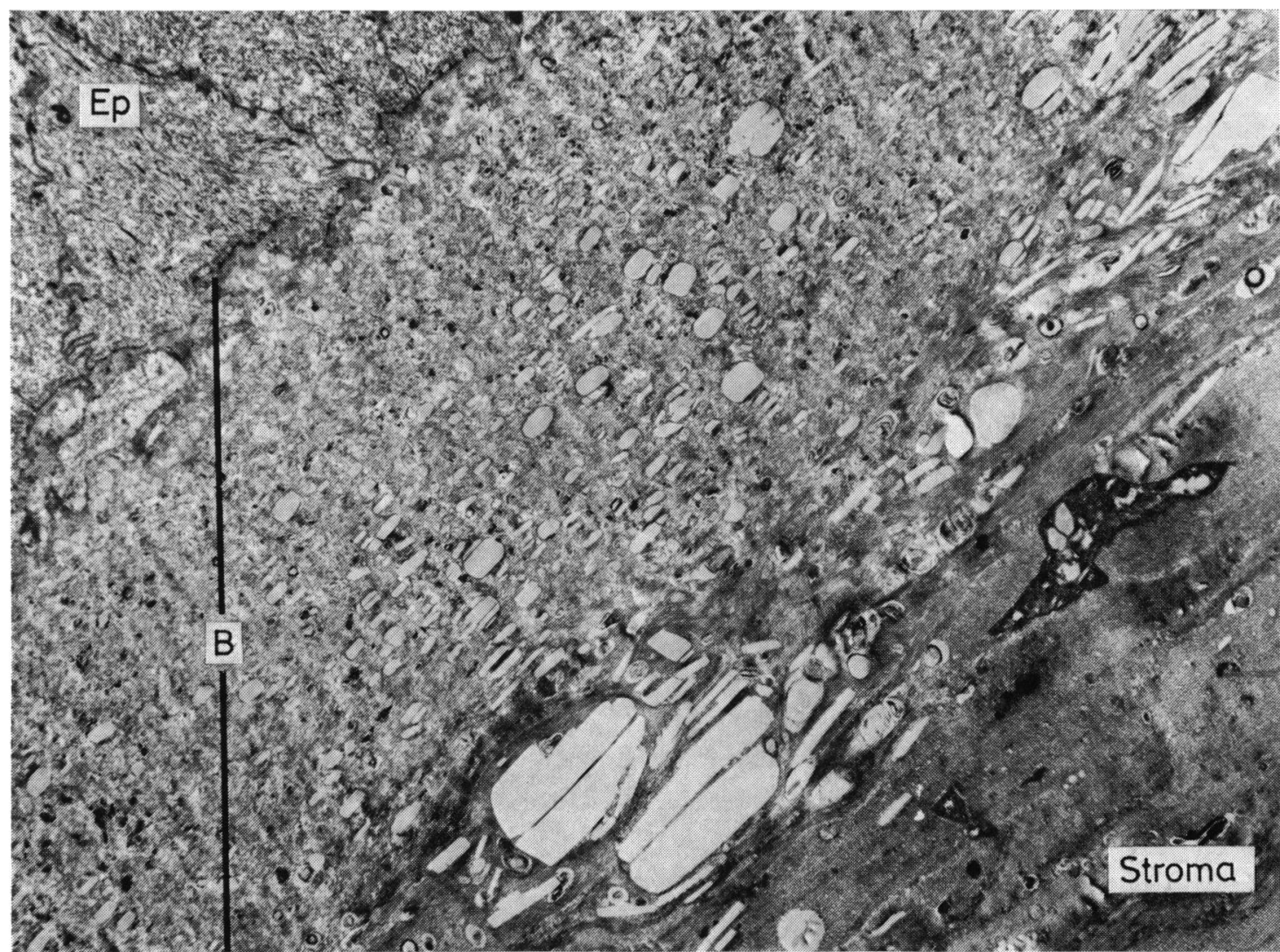

Fig. 3 Electron micrograph of Bowman's membrane (B) showing small oblong holes. The stroma contains larger angulated defects. Epithelium (Ep). $\times 6000$

corneal disease, nor is there evidence in the patient or surviving relatives of a familial disorder of lipid or carbohydrate metabolism. Some reports have stressed the high blood lipid levels found in patients with this disease, though unfortunately there is some doubt whether the levels were fasting, and often reports of blood sugar levels are missing. Standards of what constitutes hypercholesterolaemia have also changed over the years, which adds to the difficulty of interpretation. Nevertheless, Bron et al. (1972) reported very high levels of blood cholesterol and triglyceride in one patient with crystalline stromal dystrophy and moderately high levels of these lipids in a second patient. Two sisters in the same family were said to have hyperlipoproteinaemia. No figures for fasting blood sugars were given, and it is assumed that these were cases of true hyperlipoproteinaemia, though they were not classified by type. Bron et al. (1972) appear to be on less certain ground when they try to link the presence of the arcus lipidalis with the high blood cholesterol even using Forsius's criteria (Forsius, 1954). On balance it seems unlikely that such a localised anomaly of corneal stroma bears any relationship to a systemic lipid disorder. Furthermore, it is probable that a proportion of the patients with crystalline stromal corneal dystrophy will have raised blood cholesterol values, as they are relatively common in the general population.

The distinction between the generalised hyperlipidaemia and a localised disorder of lipid metabolism is important. A high serum cholesterol is a contraindication to keratoplasty, as cholesterol accumulates much more rapidly in the injured cornea of cholesterol-fed rabbits (Rodger, 1971) and in the corneal grafts of hyperlipidaemic patients (Rodger and Sinclair, 1969). In the case of stromal corneal dystrophy, where the disorder of lipid metabolism appears to be localised and unrelated to any disorder of corneal corpuscles, it is too early to decide whether corneal grafting will be successful, as this procedure has been performed on only a few patients. 


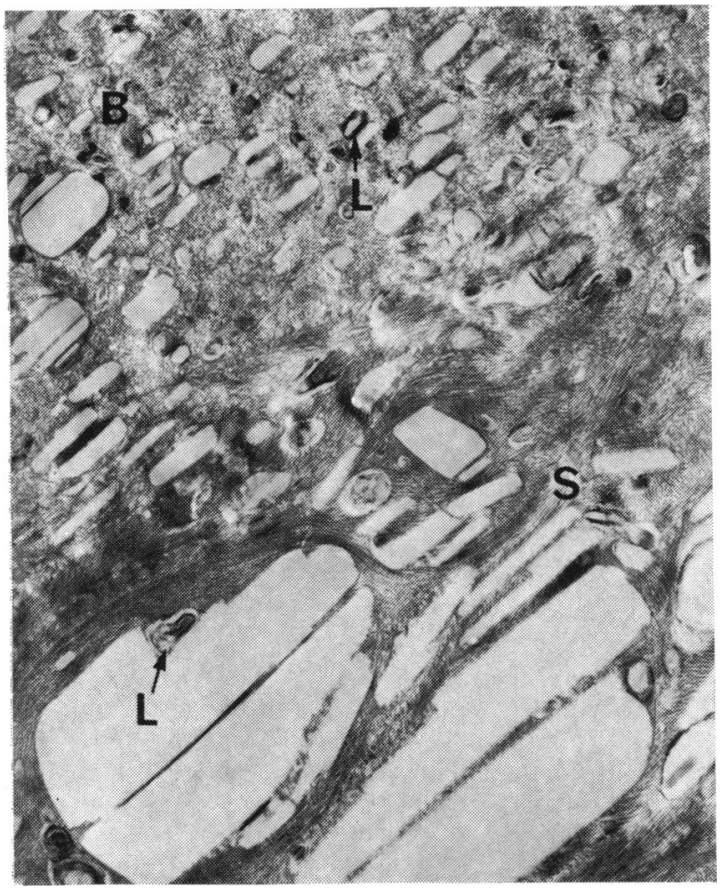

Fig. 4 Higher magnification of Fig. 3 showing disorganisation of collagen bands by the presence of angulated holes. Multilamellar bodies (L). Bowman's membrane (B). Stroma (S). $\times 10800$

Delleman and Winkelman (1968) grafted 4 patients, 2 with lamellar and 2 with penetrating grafts. The latter type was more successful, but the deposition of crystals was observed in both grafts within a year, and in 1 patient subepithelial opacity was also observed. Garner and Tripathi (1972) investigated the cytological changes in a lamellar keratoplasty obtained from 1 of the patients treated by Bron et al. (1972). They reported crystal deposition in relation to the graft despite lipid clearing therapy, but no evidence of subepithelial thickening was noted as observed by Delleman and Winkelman (1968) and in the present case. Paufique et al., (1964) reported 1 successful lamellar keratoplasty, and Ehlers and Matthiessen (1973) performed 2 penetrating keratoplasties with apparently reasonable results, though neither has supplied subsequent data on the progress of the graft. From the small number of grafts performed in crystalline stromal dystrophy it seems that the value of keratoplasty is not yet established.

The availability in the present case of a fullthickness corneal disc for histochemical and electron microscope studies has made a more complete assessment of the extent of the corneal involvement possible. As with other cases (Bonnet et al., 1934;

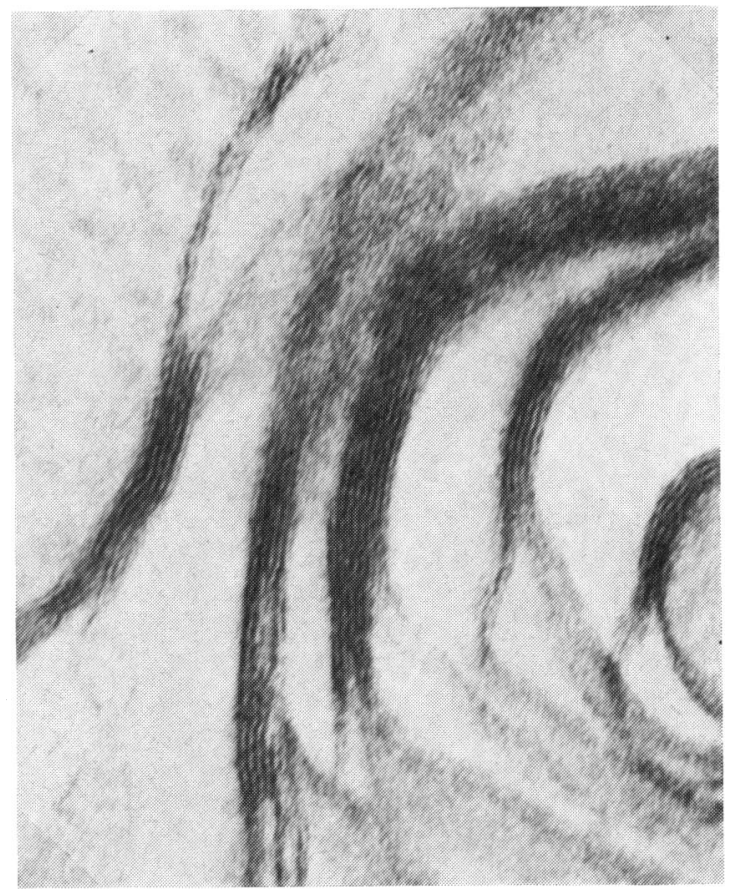

Fig. 5 Multilamellar body in corneal stroma showing 4-4.5 $\mathrm{nm}$ periodicity. $\times 172000$

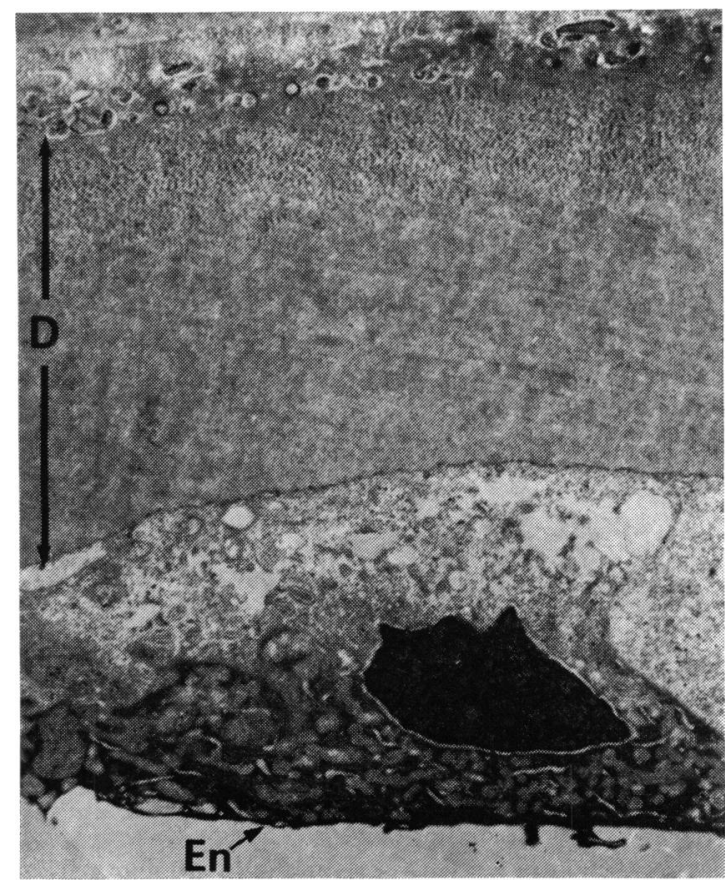

Fig. 6 Descemet's membrane (D) contains some granular material. Endothelium (En). $\times 5800$ 
Delleman and Winkelman, 1968; Garner and Tripathi, 1972; Ehlers and Matthiessen, 1973) the epithelium was unaffected. Malbran (1972), however, reported hyperplasia of the epithelium in 1 case, and Ghosh and McCulloch (1977) described foamy histiocytes in the epithelium of their case. Bowman's membrane is severely affected in most of the cases described. In the present case there were deposition of crystals and multilamellar bodies in the disrupted Bowman's membrane. Delleman and Winkelman (1968) described diffuse staining for lipids in Bowman's membrane similar to that seen in the present case. In the case described by Garner and Tripathi (1972), however, Bowman's membrane was destroyed except at the periphery of the cornea. The most severely disrupted region in the present case was in the superficial stroma just deep to Bowman's membrane, where the crystals were larger and the aggregates more compact. Lamellated bodies and lipid droplets were distributed throughout the stroma. Descemet's membrane and the endothelium appeared structurally normal apart from a vague, dark, granular material in part of Descemet's membrane.

There has been some dispute in the past about the identity of the birefringent crystals in the cornea, but the histochemical studies of Delleman and Winkelman (1978) and Garner and Tripathi (1972) suggest that they are cholesterol. The birefringent nature of the crystals and their high melting point in the present study also suggest that the crystals are cholesterol. This has been confirmed with the PAN method (Adams, 1961) for cholesterol, which is more specific than the Schultz method (Adams, 1965). Cholesterol esters form a major point of the lipid in the cornea in the present case, but no triglyceride was found by specific histochemical staining by the lipase calcium method (Adams et al., 1966) despite the suggestion by Garner and Tripathi (1972) that the droplets were triglyceride.

The lipid droplets and crystals resemble in form those found in the subepithelial region of the cornea in hypercholesterolaemic rabbits (Rodger, 1971). Similar lipid droplets have been observed in atherosclerotic plaques (Weller, 1966; Weller, 1967). From their physical properties the birefringent droplets observed in polarised light appeared to correspond to the larger lamellated droplets. The $4.4-5 \mathrm{~nm}$ periodicity of the lamellated droplets suggests that they are formed from polar lipids, probably phospholipid mixed with cholesterol.

The origin of the cholesterol and cholesterol ester deposits in the cornea is most probably from the blood via the nutrient aqueous humour. However, the reason why there is local hypercholesterolosis of the cornea in crystalline stromal dystrophy is unclear. As cholesterol and ester deposition can be induced in a similar site by hypercholesterolaemia, particularly where the cornea is scarred (Rodger, 1971), it may be due to a defect in the clearance of cholesterol from the cornea. Calcium salts similarly accumulate in the superficial stroma and Bowman's zone in other corneal lesions (Klintworth, 1977); this may also represent a defect in clearance of substances from this region of the cornea. Glycosaminoglycans within the corneal stroma may play a role in retaining cholesterol lipoproteins, as in the arterial wall, where dermatan sulphate appears to bind very low density lipoproteins and low density lipoproteins by ionic interaction (Iverius, 1973). The major sulphated glycosaminoglycan in the cornea, however, is keratin sulphate (Klintworth, 1977), and this differs from that in the arterial wall. In the absence of hypercholesterolaemia the deposition of cholesterol may be due to an abnormality in the composition of mucosubstances in the cornea itself. Histochemical stains on affected corneae have revealed no abnormality in the glycosaminoglycans (Malbran, 1972; Garner and Tripathi, 1972), but the defect may be more subtle than that detectable by histochemical techniques.

We thank Dr Olga Bayliss-High for her invaluable help with the histochemistry, and the staff of the neuropathology and electron microscope laboratories for their technical assistance. Mrs Olive Huber kindly typed the manuscript.

\section{References}

Adams, C. W. M. (1961). The perchloric acid-naphthoquinone method for the histochemical localisation of cholesterol. Nature, 192, 331-332.

Adams, C. W. M. (1965). Neurohistochemistry, p. 61. Elsevier Publishing Company: Amsterdam.

Adams, C. W. M., Abdulla, Y. H., Bayliss, O. B., and Weller, R. O. (1966). Histochemical detection of triglyceride esters with specific lipases and calcium-lead sulphide technique. Journal of Histochemistry and Cytochemistry, 14, 385-395.

Babel, J., Englest, U., and Ricci, A. (1973). Crystalline dystrophy of the cornea: histological and ultrastructural study. Archives d'Ophtalmologie, 33, 721-734.

Bonnet, P., Paufique, L., and Bonamour, G. (1934). Cristaux de cholestérine au centre de la cornée avec gérontoxon. Bulletin des Sociétés d'Ophtalmologie de France, 46, 225-229.

Bron, A. J., Williams, H. P., and Carruthers, M. E. (1972). Hereditary crystalline stromal dystrophy of Schnyder. I. Clinical features of a family with hyperlipoproteinaemia. British Journal of Ophthalmology, 56, 383-399.

Delleman, J. W., and Winkelman, J. E. (1968). Degeneratio corneae cristallineae hereditario. A chemical, genetical and histological study. Ophthalmologica, 155, 409-426.

Ehlers, N., and Matthiessen, M. E. (1973). Hereditary crystalline corneal dystrophy of Schnyder. Acta Ophthalmologica, 51, 316-324.

Forsius, H. (1954). Arcus senilis corneae. Acta Ophthalmologica, Suppl. 42, 78-107.

Garner, A., and Tripathi, R. C. (1972). Hereditary crystalline stromal dystrophy of Schnyder. II. Histopathology 
and ultrastructure. British Journal of Ophthalmology, 56, 400-408.

Ghosh, M., and McCulloch, C. (1977). Crystalline dystrophy of the cornea: a light and electron microscopic study. Canadian Journal of Ophthalmology, 12, 321-329.

Gillespie, F. D., and Covelli, B. (1963). Crystalline corneal dystrophy. American Journal of Ophthalmology, 56, 465467.

Iverius, P-H. (1973). Possible role of the glycosaminoglycans in the genesis of atherosclerosis. In Atherogenesis: Initiating Factors. Ciba Foundation Symposium 12, pp. 185-196. Elsevier: Amsterdam.

Klintworth, G. K. (1977). The cornea-structure and macromolecules in health and disease. A Review. American Journal of Pathology, 89, 719-808.

Malbran, E. S. (1972). Corneal dystrophies: a clinical, pathological and surgical approach. American Journal of Ophthalmology, 74, 771-809.
Paufique, L., Ravault, M. P., Bonnet, M., and Laurent, C. (1964). Schnyder's crystalline dystrophy. Bulletin des Sociétés d'Ophtalmologie de France, 64, 104-107.

Rodger, F. C. (1971). A study of the ultrastructure and cytochemistry of lipid accumulation and clearance in cholesterol-fed rabbit cornea. Experimental Eye Research, 12, 88-93.

Rodger, F. C., and Sinclair, H. M. (1969). Metabolic and Nutritional Eye Diseases, p. 143. C. C. Thomas: Springfield, Illinois.

Schnabel, R. (1964). Eine topochemische Methode zur Differenzierung des freien und veresterten Cholesterins. Acta Histochemica, 18, 161-167.

Weller, R. O. (1966). The ultrastructure of intracellular lipid accumulations in atheroma. Journal of Atherosclerosis Research, 6, 184-189.

Weller, R. O. (1967). Cytochemistry of lipids in atherosclerosis. Journal of Pathology and Bacteriology, 94, 171-182. 